\title{
DISCOVERY OF A PROBABLE PHYSICAL TRIPLE QUASAR ${ }^{1}$
}

\author{
S. G. Djorgovski, ${ }^{2,3}$ F. Courbin, ${ }^{3}$ G. Meylan, ${ }^{3}$ D. Sluse, ${ }^{3}$ D. Thompson, ${ }^{4}$ A. Mahabal, ${ }^{2}$ And E. Glikman ${ }^{2}$ \\ Received 2006 December 22; accepted 2007 April 25; published 2007 May 22
}

\begin{abstract}
We report the discovery of the first known probable case of a physical triple quasar. A previously known double system, QQ 1429-008 at $z=2.076$, is shown to contain a third, fainter QSO component at the same redshift. Deep optical and IR imaging has failed to reveal a plausible lensing galaxy group or a cluster, and we are unable to construct any viable lensing model that could lead to the observed distribution of source positions and relative intensities of the three QSO image components. There are also hints of differences in the broadband spectral energy distributions of the different components, which are more naturally understood if they are physically distinct active galactic nuclei (AGNs). Therefore, we conclude that this system is most likely a physical triple quasar, the first such close QSO grouping known at any redshift. The projected component separations are $\sim 30$ $50 \mathrm{kpc}$, typical of interacting galaxy systems. The existence of this highly unusual system supports the standard picture in which galaxy interactions lead to the onset of QSO activity.
\end{abstract}

Subject headings: galaxies: interactions — gravitational lensing — quasars: general

\section{INTRODUCTION}

It is now generally accepted that the onset and fueling of AGN activity are closely related to dissipative tidal interactions and mergers of galaxies, and that there is a close synergy between the formation and growth of supermassive black holes (SMBHs) and their host galaxies. For recent reviews and further references, see, e.g., the proceedings edited by Ho (2004) or the study by Hopkins et al. (2006).

If galaxy interactions are responsible for triggering of QSO activity, it is reasonable to expect that in some cases, this would occur in both components of an interacting galaxy pair, resulting in a binary QSO. Starting from the early discoveries of such systems (Djorgovski et al. 1987; Meylan et al. 1990), there are now many tens, if not hundreds, of physical binary QSOs known (Hennawi et al. 2006; Myers et al. 2007). Their frequency may be up to 2 orders of magnitude higher than what may be expected from simple extrapolations of galaxy clustering at comoving scales $<100 \mathrm{kpc}$, which can be understood if interactions enhance the probability of QSO activity, atop of the normal clustering of their host galaxies (Djorgovski 1991; Kochanek et al. 1999; Hennawi et al. 2006). Sometimes there are ambiguities between the binary QSO and gravitational lensing interpretations of close QSO pairs; see, e.g., Kochanek et al. (1999) or Mortlock et al. (1999) for discussions and references.

One such case is the QSO pair B1429-008 (= J1432-0106; Hewett et al. 1989), whose brighter component was originally found in the Large Bright QSO Survey (LBQS; Hewett et al. 1991). The two brightest QSO components are at $z=2.076$ and are separated by 5.14", corresponding to a projected separation of $43 \mathrm{kpc}$ (proper) or $132 \mathrm{kpc}$ (comoving); we use the

\footnotetext{
${ }^{1}$ Based in part on the data obtained at the W. M. Keck Observatory, a scientific partnership among the California Institue of Technology, the University of California, and NASA, made possible by the generous financial support of the W. M. Keck Foundation; data obtained with VLT/Antu telescope at ESO-Paranal Observatory; and data obtained with the Hubble Space Telescope (HST), operated by NASA. This research also made use of data obtained from the US National Virtual Observatory, which is sponsored by the NSF.

${ }^{2}$ Division of Physics, Mathematics, and Astronomy, California Institute of Technology, Pasadena, CA 91125.

${ }^{3}$ Laboratoire d'Astrophysique, Ecole Polytechnique Fédérale de Lausanne (EPFL), CH-1290 Sauverny, Switzerland.

${ }^{4}$ Large Binocular Telecope Observatory, University of Arizona, Tucson, AZ 85721 .
}

standard Friedmann-Lemaitre cosmology with $h=0.7$, $\Omega_{m}=0.3$, and $\Omega_{\Lambda}=0.7$ throughout.

In this Letter we report on the discovery of a third QSO component in this system and discuss additional evidence against the gravitational lensing hypothesis. We then conclude that this is most likely the first known case of a physical triple QSO, which we denote as QQQ 1432-0106 below.

\section{THE DATA AND THE OBSERVATIONS}

We obtained images of the field in the Cousins $I$ band, using the Low-Resolution Imaging Spectrometer (LRIS; Oke et al. 1995) at the W. M. Keck Observatory's 10 m telescope on 1994 April 13 UT, in good conditions. Eight exposures of $300 \mathrm{~s}$ each were obtained and processed using standard techniques. A section of the co-added image is shown in Figure 1. Several faint sources surround the previously known QSO pair. We also obtained $K$-band images using the Near-Infrared Camera (NIRC) on Keck (Matthews \& Soifer 1994) on 1994 April 6 UT and again on 1995 June 15 UT; the total usable exposure time was $3360 \mathrm{~s}$. For the purposes of the present analysis, we co-added these data with the ESO VLT $K_{s}$-band images described by Faure et al. (2003). The central portion of the stacked image is shown in Figure 1. We use the same notation for the fainter sources as in Faure et al. (2003), except for their source 5, which we label as $\mathrm{C}$. The limiting magnitudes $(1 \sigma)$ for the stacked $K$-band image are 24.80, 24.05, and 23.30 mag for the apertures of $1^{\prime \prime}, 2^{\prime \prime}$, and 4 " diameter, respectively.

Long-slit spectra were obtained using the LRIS on 1995 February 1 , in good conditions. A single 300 s exposure was obtained for the QSO component A, and two 1200 s exposures capturing the components B, C, 3, and 8 were obtained with a slit position angle P.A. $=69^{\circ}$. We used a 300 line $\mathrm{mm}^{-1}$ grating and a $1.5^{\prime \prime}-$ wide slit, giving a mean dispersion of $\sim 2.49 \AA$ pixel $^{-1}$ and a spectroscopic resolution of FWHM $\sim 17 \AA$, with a wavelength coverage from $\sim 3850$ to $\sim 8890 \AA$. The data were reduced using standard techniques. The spectra of the components A, B, and $\mathrm{C}$ are shown in Figure 2; it is evident that $\mathrm{C}$ is a QSO component at the same redshift as A and B. No redshifts could be obtained for the faint objects (probably galaxies) 3 and 8, but they show no indications of AGNs in their spectra.

The J2000 coordinates of the QSO A, taken as a mean of the USNO A2 and B1 catalogs, are $\alpha=14^{\mathrm{h}} 32^{\mathrm{m}} 29.232^{\mathrm{s}}, \delta=$ 

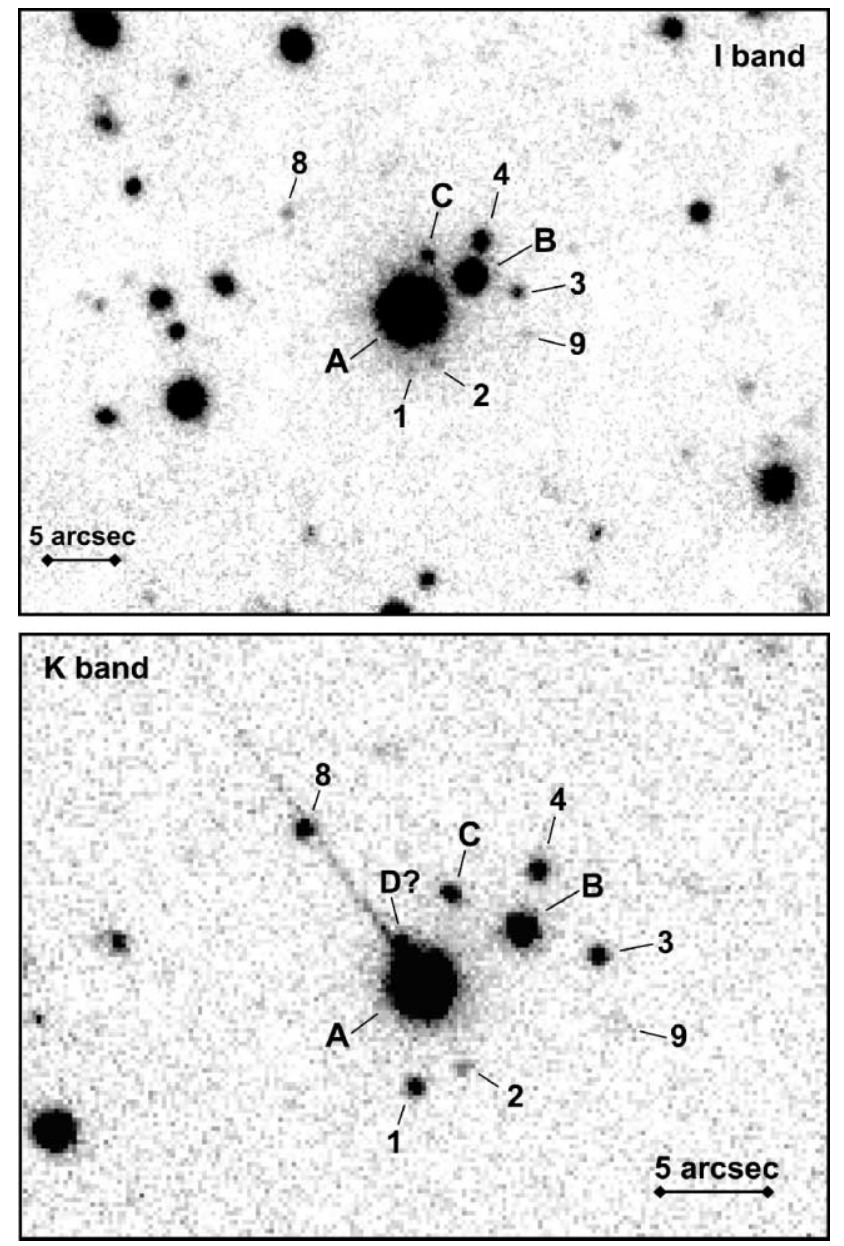

FIG. 1.-Top: Keck I-band image of the field, with the QSO components $\mathrm{A}, \mathrm{B}$, and $\mathrm{C}$ and other faint objects nearby labeled; the numbers follow and extend the notation from Faure et al. (2003). Bottom: Sum of the Keck and VLT $K$-band images of the field. The diagonal streak is a charge transfer artifact from the Keck images, prior to their rotation to the cardinal orientation. North is up, east is to the left, and the scale is given by the inset bars.

$-01^{\circ} 06^{\prime} 15.68^{\prime \prime}$. The offsets to component B are $\Delta \alpha=4.46^{\prime \prime}$ west and $\Delta \delta=2.49^{\prime \prime}$ north, and those to component $\mathrm{C}$ are $\Delta \alpha=1.22^{\prime \prime}$ west and $\Delta \delta=4.11^{\prime \prime}$ north, taken from Faure et al. (2003). They correspond to the projected separations AB, A-C, and B-C of 5.11", 4.29", and 3.62", respectively, or 43,36 , and 30 proper kpc (appropriate for the gravitationally bound systems), or 131, 110, and 93 comoving kpc.

Table 1 lists the photometric data for the three QSOs. The $R J H_{\mathrm{F} 160 \mathrm{w}} K_{s}$ magnitudes are from Faure et al. (2003), the SDSS ugi magnitudes are from Hennawi et al. (2006) and Myers et al. (2007), and we used their measurements of the QSO B and our Keck $I$ band data to infer the $i$-band magnitude of the QSO C. The implied colors for the QSOs A and B show modest but statistically significant differences. However, the optical-to-IR colors of the QSO $\mathrm{C}$ are significantly redder: the $\left(R-K_{s}\right)$ colors are $2.49 \pm 0.03,2.27 \pm 0.03$, and $3.23 \pm 0.21$ for the

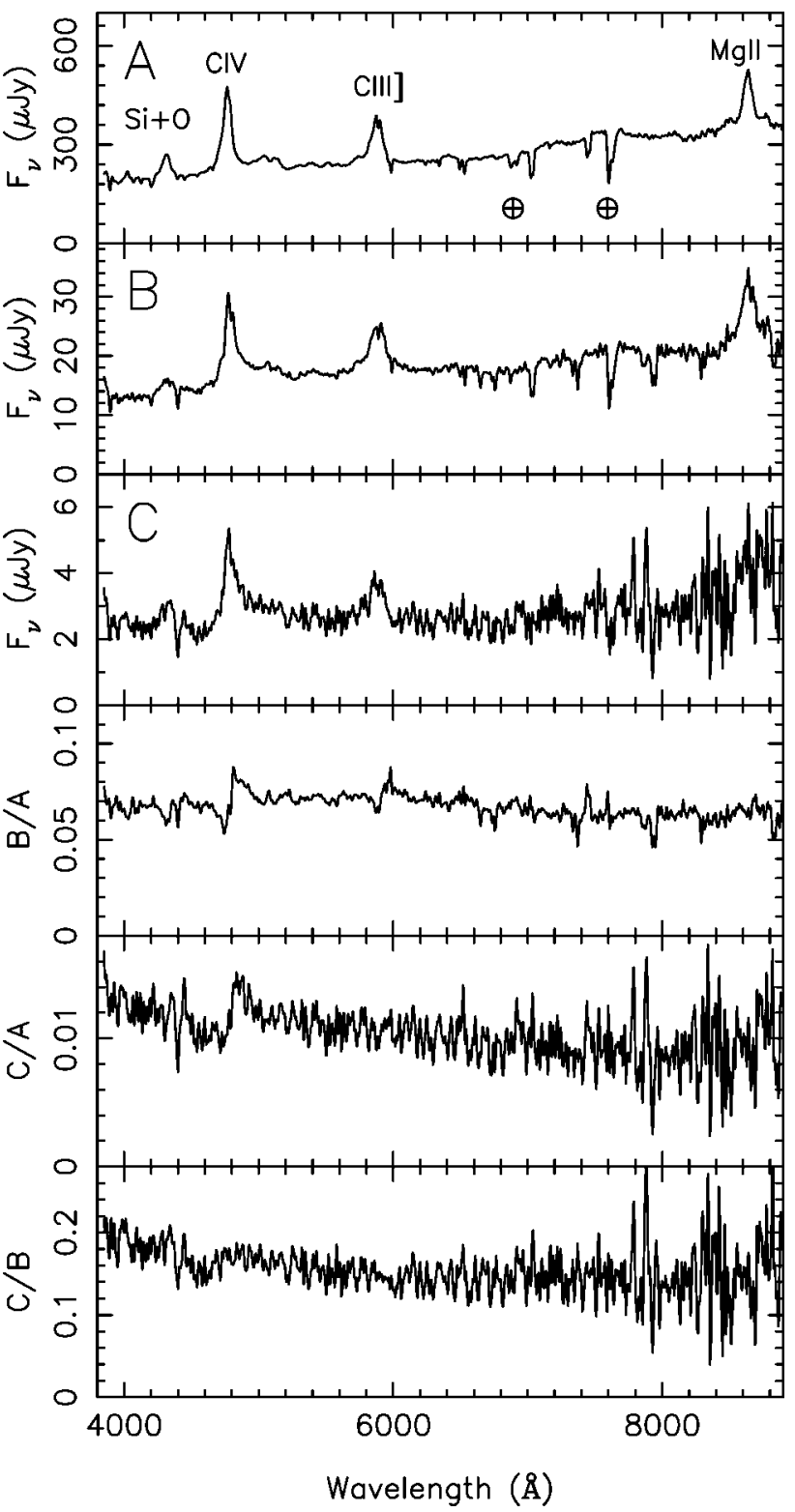

FIG. 2.- Spectra of the QSO components A, B, and C (top three panels), and their ratios (bottom three panels), obtained at the Keck. The prominent emission lines, Si IV + O IV $\lambda 1400$, C IV $\lambda 1549$, C III] $\lambda 1909$, and $\mathrm{Mg}$ II $\lambda 2799$, are labeled, as well as the atmospheric absorption A and B bands. A number of metallic absorption systems are detected; see the text for more details. The bottom three panels show the ratios of the spectra.

components $\mathrm{A}, \mathrm{B}$, and $\mathrm{C}$ respectively; the $(R-J)$ colors are $1.13 \pm 0.03,0.85 \pm 0.03$, and $1.87 \pm 0.13$; and the $\left(i-K_{s}\right)$ colors are 2.44, 2.16, and $3.3 \pm 0.3$. We note that the Balmer break is redshifted to be between the optical and IR bands, so one possible interpretation of the data is that there is a significant starlight component in the measured IR flux from component $\mathrm{C}$, which is the faintest $\mathrm{AGN}$ of the three.

While the optical flux ratio $\mathrm{A} / \mathrm{B}$ is $25 \pm 3$, in the $\mathrm{X}$-rays

TABLE 1

Magnitudes For the Three QSO Components

\begin{tabular}{lccccccc}
\hline \hline QSO & $R$ & $J$ & $H_{\mathrm{F} 160 \mathrm{~W}}$ & $K_{s}$ & $u$ & $g$ & $i$ \\
\hline A $\ldots \ldots$ & $17.45 \pm 0.02$ & $16.32 \pm 0.01$ & $15.88 \pm 0.01$ & $14.96 \pm 0.01$ & 17.88 & 17.81 & 17.48 \\
B $\ldots \ldots$ & $20.85 \pm 0.02$ & $20.00 \pm 0.01$ & $19.27 \pm 0.01$ & $18.58 \pm 0.01$ & 21.40 & 21.08 & 20.82 \\
C $\ldots \ldots$ & $23.53 \pm 0.06$ & $21.66 \pm 0.07$ & $21.17 \pm 0.04$ & $20.30 \pm 0.15$ & $\ldots$ & $\ldots$ & $23.7 \pm 0.2$ \\
\hline
\end{tabular}


this flux ratio is significantly smaller, $5.3 \pm 1.8$, on the basis of ChaMP measurements (Kim et al. 2007). We also note that the QSO A is detected by FIRST at $S_{1.4 \mathrm{GHz}}=0.93 \pm 0.16 \mathrm{mJy}$, but QSO B is below their detection limit; deeper radio observations would be very useful.

Figure 2 also shows the ratios of the three spectra. The spectra of components A and B are broadly similar, and we note that Mortlock et al. (1999) found that the observed spectroscopic differences between components A and B are typical for a random pair of QSOs at these redshifts. However, component $\mathrm{C}$ has a significantly bluer continuum in the observed visible regime, which is the reverse of the trend given by its optical-to-IR colors. This essentially eliminates the possibility that it is made dimmer and redder by extinction due to a foreground galaxy. The line C IV $\lambda 1549$ in components B and C clearly has a different shape from that in component $\mathrm{A}$, and possibly some of the other broad lines are different as well.

We used the cross-correlation technique as implemented in IRAF to measure the rest-frame velocity shifts between the QSO spectra. From the peak of the correlation function, we get $\Delta V_{A B}=280 \pm 160 \mathrm{~km} \mathrm{~s}^{-1}$, consistent with the original measurements by Hewett et al. (1989), and $\Delta V_{B C}=100 \pm$ $400 \mathrm{~km} \mathrm{~s}^{-1}$, consistent both with zero and with the relative velocities typical for the bound or interacting galaxies.

Several prominent absorption systems are seen, including lines of the $C$ IV doublet $\lambda 1548.195, \lambda 1550.770$; Al II $\lambda 1670.787$; Fe II $\lambda 2367.591, \lambda 2382.765, \lambda 2586.650, \lambda 2600.173$; and $\mathrm{Mg}$ II doublet $\lambda 2796.352, \lambda 2803.531$. In the QSO A, the two strongest absorbers are at $z_{\text {abs, } A 1}=1.5115$ and $z_{\text {abs, } A 2}=1.6617$; in the QSO B, they are at $z_{\mathrm{abs}, B 1}=1.8366$ and $z_{\mathrm{abs}, B 2}=1.513$; and a strong $\mathrm{C}$ IV absorber is seen in the spectrum of the QSO C at $z_{\text {abs, } C 1}=1.840$. Presumably the absorbers A1 and B2 and the absorbers $\mathrm{B} 1$ and $\mathrm{C} 1$ are caused by the same intervening galaxies. Some of the faint galaxies numbered in Figure 1 are probably responsible for these foreground systems.

We performed image deconvolutions of the data, using the MCS algorithm (Magain et al. 1998). The most reliable one, in terms of the independently constrained point-spread function, requires using the VLT $R$-band data, shown in Figure 3. QSO images A and B are the only ones consistent with being unresolved, and an extended component is possible for component C. Its total magnitude, measured in a $2^{\prime \prime}$ aperture, is $R_{C}=23.47 \pm 0.2 \mathrm{mag}$, and the flux is divided roughly equally between the unresolved AGN component $\left(R_{C \text {, unres }}=24.3 \pm\right.$ $0.2 \mathrm{mag}$ ) and a resolved component, presumably a host galaxy $\left(R_{C \text {, host }}=24.2 \pm 0.2 \mathrm{mag}\right)$. Deconvolution also reveals a significant extended component to the south-southwest of QSO A, suggestive of a tidally distorted host galaxy.

While the differences in the broadband spectral energy distributions of the three QSO components could be, in principle, explained away in the context of gravitational lensing with the usual invocations of variability and time delays, a more natural explanation is that the three components are physically distinct AGNs.

\section{THE LENS HYPOTHESIS: MODELING}

Faure et al. (2003) presented the most comprehensive analysis to date for this system, while being unaware of the QSO nature of component C. Discovery of this new QSO component, its flux ratios relative to components $\mathrm{A}$ and $\mathrm{B}$, and the greater depth of the Keck+VLT data provide significant new constraints on the lensing hypothesis.

A triply imaged QSO usually consists of two images plus one central image. In most cases, this central image is not

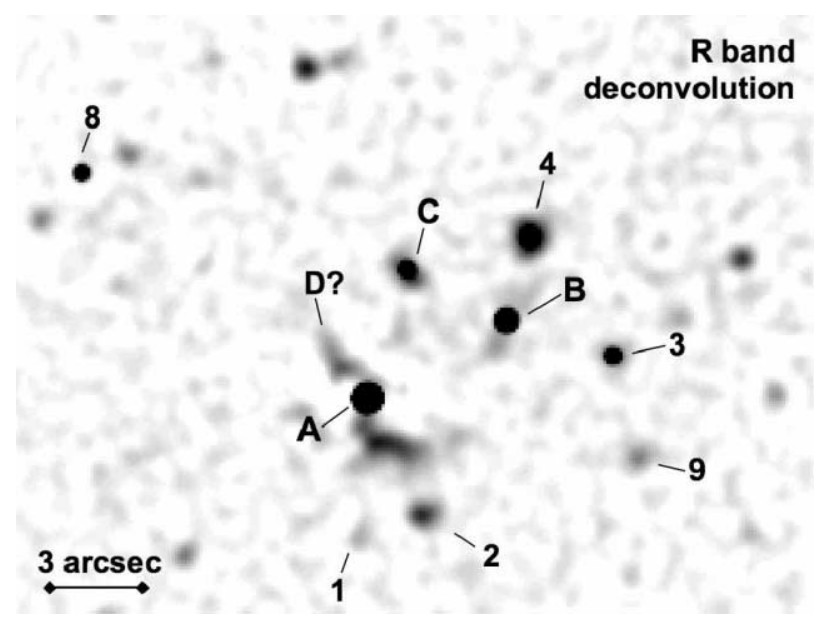

FIG. 3.-Image deconvolution of the VLT $R$-band image. The notation is the same as in Fig. 1. Note the extended, resolved structure south-southwest of QSO A, suggestive of a tidally distorted galaxy; the possible object D may be a part of it. Hints of the resolved host galaxies are also seen underlying QSOs B and C.

visible, except in APM 08729+5255 (Lewis et al. 2002) and PMNJ 1632-0033 (Winn et al. 2003). The geometry of the three components in QQQ 1432-0106 is obviously different: the three images form a triangle on the plane of the sky. Instead, we could be observing a quadruply imaged QSO, and, if so, we would need to find a candidate for the fourth image or to consider the possibility that two images are merged like in MG 2016+112 (Lawrence et al. 1984; Koopmans et al. 2002).

We investigate two lensing scenarios. In model L1, we assume that object D is the fourth QSO image. There is no other object in the vicinity of QQQ 1432-0106 that is not immediately ruled out as an extra lensed image due to its position relative to $\mathrm{A}$, $\mathrm{B}$, and $\mathrm{C}$ or on the basis of our Keck spectra. In model L2, we assume that QSO A is a narrow-separation, highly magnified blend of two images. We model the lensing galaxy potential as a singular isothermal sphere (SIS) with an external shear $\gamma$ added $(\mathrm{SIS}+\gamma)$. This type of model fits well the image configuration of most known simple gravitationally lensed QSOs. It is the most realistic model that we can consider given our limited number of observational constraints: it offers 1 degree of freedom when fitting a quadruply imaged QSO with unknown lensing galaxy position. Quadruple systems have eight observational constraints (the four positions of the lensed QSO images), while the model has seven parameters: the total mass of the lens, the position of the source relative to the lens, the amplitude $\gamma$ and P.A. $\theta_{\gamma}$ of the shear, and the lensing galaxy position.

We compute lens models using the GRAVLENS software (Keeton 2001). ${ }^{5}$ As the position of the lensing galaxy is completely unknown, we adopt a twofold strategy. First, we explore the parameter space by fitting models with fixed $\theta_{\gamma}$ and galaxy positions, scanning the $2^{\prime \prime}$ region around the barycenter of quasars A, B, and C. For each galaxy position, a broad range of $\theta_{\gamma}$ is explored. We then select the model with the best $\chi^{2}$, adopt its lens center and shear P.A., and run GRAVLENS, with all the model parameters allowed to vary. For scenario L1, we adopt $0.02^{\prime \prime}$ relative astrometric errors. For scenario L2, we split image A into two subcomponents, A1 and A2, separated by $0.05^{\prime \prime}$. HST images analyzed by Faure et al. (2003) imply that A cannot be a blend wider than $0.05^{\prime \prime}$. For A1 and A2, we artificially increase the error bars up to $0.2^{\prime \prime}$, in order to

${ }^{5}$ Software available at http://redfive.rutgers.edu/ keeton/gravlens/. 
reflect the poor astrometric constraint on the putative components of the blend. Therefore, GRAVLENS is relatively free to recover the image positions within loose error boxes.

We do not get acceptable fits with either model. In the L1 scenario, our best model predicts lensed images typically $0.5^{\prime \prime}$ away from the observed positions as indicated by the large value of the reduced $\chi^{2}=1941$. Such a model also predicts image $\mathrm{D}$ to be the brightest, and images $\mathrm{B}$ and $\mathrm{C}$ to have similar brightness, in a clear contradiction with the observations. In scenario L2, our best model has $\chi^{2}=74$. Images B and C are fairly well reproduced, but the two merging images A1-A2 are predicted to lie about $1.2^{\prime \prime}$ away from their observed positions, and we can thus reject this possibility as well. Thus, simple lens models do not provide acceptable fits of the observed lensed image configuration. Better fits to the data would require the addition of a secondary lens, resulting in a model with zero degrees of freedom. Likewise, if we introduced an elliptical lens, we would add two new parameters, which would be hard to constrain. With a simple SIS $+\gamma$ model, we therefore already reach the limit of available constraints.

We estimate the total mass of the lens inside the Einstein radius for our best model L2. It can be approximated as a function of redshift as $M_{\mathrm{E}}=\left(2 \times 10^{12} z_{\text {lens }}\right) M_{\odot}$ up to $z=0.8$, and as $M_{\mathrm{E}}=\left(5 \times 10^{12} z_{\text {lens }}-2.5 \times 10^{12}\right) M_{\odot}$ for $0.8<z<1.4$. The shear direction for this model is P.A. $=+102^{\circ}$, and its amplitude is $\gamma=0.19$. In contrast to MG 2016+112, no lensing galaxy is seen in QQQ $1432-0106$, down to $K=23.3$ ( $1 \sigma$ limit in a $4^{\prime \prime}$ aperture). In MG 2016+112, the lens is at $z=1.01$ and has $H=18.5 \mathrm{mag}$. The mass of the putative lens, if placed at an overly optimistic $z=0.5$, is $M_{\mathrm{E}}=10^{12} M_{\odot}$, in a $4^{\prime \prime}$ aperture, using model L2. This corresponds to a velocity dispersion of $\sigma=330 \mathrm{~km} \mathrm{~s}^{-1}$. If the lens is at $z=1.4$, then $\sigma=580 \mathrm{~km} \mathrm{~s}^{-1}$. If the lens is at $z=0.5$, then its absolute magnitude would be $M_{K}=-25.6$ using the Faber-Jackson relation ( $\sigma$ from lens model, without conversion from $\sigma_{\text {lens }}$ to $\left.\sigma_{\text {dyn }}\right)$, and $M_{\star}(K)=-23.9$ (Gardner et al. 1997). If it is at $z=1.4$, then $M_{K}=-28.0$. Using $K$-corrections from Mannucci et al. (2001), this translates to apparent magnitudes of $K=17.4$ and $K=17.6 \mathrm{mag}$, respectively. The presence of such lensing galaxies is strongly excluded by our deep $K$-band images, even if they were to be aligned with the brightest QSO component (which would be a very difficult geometry to arrange).

We are thus unable to produce any plausible lensing model that would account for the observed geometry and intensity ratios of the QSO images, and moreover we have strong upper limits on the existence of necessary lensing galaxies or groups. We also note that Faure et al. (2003) have placed strong limits on weak-lensing distortions in this field, which would be expected if a massive lens was present, regardless of its obscuration. Therefore, we conclude that this system is highly unlikely to be a case of gravitational lensing.

\section{DISCUSSION AND CONCLUSIONS}

The observed differences in the spectra and colors of the QSO components, and the great difficulty in modeling the sys- tem as a gravitational lens, strongly suggest that we are dealing with a case of a physical triple QSO. The projected separations are typical of those seen in galaxy interactions.

Measurements of a QSO three-point correlation function $\zeta(r)$ at such physical separations are currently not available, at any redshift. However, we can make a rough estimate as follows, using the traditional hierarchical clustering Ansatz, $\zeta_{123}(r)=Q\left[\xi_{12}(r)+\xi_{13}(r)+\xi_{23}(r)\right]$, where $Q \approx 1$ and where $\xi_{i j}(r)$ are the amplitudes of the pairwise two-point correlation function for the three components (Peebles 1993). From Hennawi et al. (2006), we get the projected two-point correlation function $w(r) \approx 100$ for $r \sim 20-30 h^{-1}$ proper kpc, the observed separations in this triplet; and this is at least an order of magnitude stronger clustering than for galaxies at comparable redshifts (Djorgovski 1991; Kochanek et al. 1999; Hennawi et al. 2006). Assuming the same average projection effects, we get the estimate of $\zeta \sim 300$ for this triplet. These are the "excess probabilities" over the uniform random distribution. Integrating the QSO luminosity function at $z \sim 2$ from Richards et al. (2006), for the observed absolute magnitudes of these QSOs, we derive the probabilities of finding QSO B so close to QSO $\mathrm{A}, p_{A B}=6 \times 10^{-8}$, and of the QSOs C and A, $p_{A C}=8 \times$ $10^{-8}$, if QSOs were randomly distributed in space. For all three QSOs to be found so close, $p_{A B C}=5 \times 10^{-15}$. Thus, clearly this triplet is not there by random chance, and just as with the observed abundance of QSO binaries, this excess can be naturally understood as a consequence of the enhanced propensity for fueling of AGNs in dissipative galaxy interactions.

Indeed, we observe QQQ 1432-0106 at a redshift where the merging activity and the comoving density of QSOs were roughly at their peak. Several components of the system can then be interpreted as a compact galaxy group caught in the process of interacting, with AGN activity ignited simultaneously in three of the participating host galaxies. Hierarchical merging in such systems could also lead to the formation of triple SMBH systems and to a number of interesting effects; see, e.g., Hoffman $\&$ Loeb (2007) for a discussion and references.

As the census of such triple-QSO systems (and the binary QSOs themselves) grows with the future observations, and the selection effects are better understood, we will have a new observational probe of the processes of galaxy-SMBH coevolution at high redshifts.

We are grateful to the staff of the W. M. Keck and ESO-VLT observatories for their expert assistance in the course of our observing runs, and to M. Pahre with assistance from some of the Keck observations. The Keck data used in this Letter were obtained in a collaborative project involving J. Cohen, B. T. Soifer, R. Blandford, G. Neugebauer, and I. Smail. S. G. D., A. M., and E. G. acknowledge partial support from NSF grant AST-0407448 and from the Ajax Foundation. F. C., G. M., and D. S. are financially supported by the Swiss National Science Foundation (SNSF). S. G. D. acknowledges the hospitality of EPFL and the Geneva Observatory, where some of this work was performed.

\section{REFERENCES}

Djorgovski, S. 1991, in ASP Conf. Ser. 21, The Space Distribution of Quasars, ed. D. Crampton (San Francisco: ASP), 349

Djorgovski, S. G., Perley, R., Meylan, G., \& McCarthy, P. 1987, ApJ, 321, L17

Faure, C., Alloin, D., Gras, S., Courbin, F., Kneib, J.-P., \& Hudelot, P. 2003, A\&A, 405, 415

Gardner, J., Sharples, R., Frenk, C., \& Carrasco, B. 1997, ApJ, 480, L99

Hennawi, J. F., et al. 2006, AJ, 131, 1
Hewett, P. C., Webster, R. L., Harding, M. E., Jedrzejewski, R. J., Foltz, C. B., Chaffee, F. H., Irwin, M. J., \& LeFèvre, O. 1989, ApJ, 346, L61

Hewett, P. C., Foltz, C. B., Chaffee, F. H., Francis, P. J., Weymann, R. J., Morris, S. L., Anderson, S. F., \& MacAlpine, G. M. 1991, AJ, 101, 1121

Ho, L., ed. 2004, Coevolution of Black Holes and Galaxies (Cambridge: Cambridge Univ. Press)

Hoffman, L., \& Loeb, A. 2007, MNRAS, 377, 957 
Hopkins, P. F., Hernquist, L., Cox, T. J., Di Mateo, T., Robertson, B., \& Springel, V. 2006, ApJS, 163, 1

Keeton, C. R. 2001, preprint (astro-ph/0102340)

Kim, M., et al. 2007, ApJS, 169, 401

Kochanek, C., Falco, E., \& Muñoz, J. 1999, ApJ, 510, 590

Koopmans, L., Garrett, M., Blandford, R., Lawrence, C., Patnaik, A., \& Porcas, R. 2002, MNRAS, 334, 39

Lawrence, C. R., Schneider, D. P., Schmidet, M., Bennett, C. L., Hewitt, J. N., Burke, B. F., Turner, E. L., \& Gunn, J. E. 1984, Science, 223, 46

Lewis, G., Ibata, R., Ellison, S., Aracil, B., Petitjean, P., Pettini, M., \& Srianand, R. 2002, MNRAS, 334, L7

Magain, P., Courbin, F., \& Sohy, S. 1998, ApJ, 494, 472

Mannucci, F., Basile, F., Poggianti, B., Cimatti, A., Daddi, E., Pozzetti, L., \& Vanzi, L. 2001, MNRAS, 326, 745
Matthews, K., \& Soifer, B.T. 1994, in Infrared Astronomy with Arrays, ed. I. McLean (Dordrecht: Kluwer), 239

Meylan, G., Djorgovski, S.G., Weir, N., \& Shaver, P. 1990, in Gravitational Lensing, ed. Y. Mellier et al. (Lecture Notes in Phys. 360 (Berlin: Springer), 111

Mortlock, D., Webster, R., \& Francis, P. 1999, MNRAS, 309, 836

Myers, A., Brunner, R., Richards, G., Nichol, R., Schneider, D., \& Bahcall, N. 2007, ApJ, 658, 99

Oke, J. B., et al. 1995, PASP, 107, 375

Peebles, P. J. E. 1993, Principles of Physical Cosmology (Princeton: Princeton Univ. Press)

Richards, G. T., et al. 2006, AJ, 131, 2766

Winn, J., Rusin, D., \& Kochanek, C. 2003, ApJ, 587, 80 